\title{
UNIFORMIZATION OF CANTOR SETS WITH BOUNDED GEOMETRY
}

\author{
VYRON VELLIS
}

\begin{abstract}
In this note we provide a quasisymmetric taming of uniformly perfect and uniformly disconnected sets that generalizes a result of MacManus [Rev. Mat. Iberoamericana 15 (1999), pp. 267-277] from 2 to higher dimensions. In particular, we show that a compact subset of $\mathbb{R}^{n}$ is uniformly perfect and uniformly disconnected if and only if it is ambiently quasiconformal to the standard Cantor set $\mathcal{C}$ in $\mathbb{R}^{n+1}$.
\end{abstract}

\section{INTRODUCTION}

The (quasisymmetric) uniformization problem asks for necessary and sufficient conditions under which a metric space $X$ is quasisymmetrically homeomorphic to a "standard" space $X_{0}$. Roughly speaking, quasisymmetric homeomorphisms are a generalization of conformal maps which preserve relative distances; see $\$ 2$ for precise definitions. The uniformization problem has been extensively studied in literature for a variety of "standard" spaces: the unit circle $\mathbb{S}^{1}[20$, the unit sphere $\mathbb{S}^{2}[3$, and geodesic trees [4. See also [2] for a general overview.

Some of the most simple metric spaces, from a topological point of view, are Cantor sets, i.e., homeomorphic images of the standard ternary Cantor set $\mathcal{C}$. Brouwer's topological characterization of Cantor sets [13, Theorem 7.4] states that a metric space is a Cantor set if and only if it is compact, perfect, and totally disconnected.

In [6], David and Semmes solved the uniformization problem in the case that the standard space $X_{0}$ is $\mathcal{C}$. Contrary to Brouwer's uniformization, in the quasisymmetric case one has to assume some quantitative versions of perfectness and total disconnectedness.

A closed nondegenerate metric space $X$ is called uniformly perfect if there exists a constant $c \geq 1$ such that for all $x \in X$ and all $r \in(0$, diam $X)$, there exists a point in $B(x, r) \backslash B(x, r / c)$. Every uniformly perfect space is perfect; on the other hand, the planar set $[0,1] \times \bigcup_{n=1}^{\infty}\{n !\}$ is perfect but not uniformly perfect.

A metric space $X$ is uniformly disconnected if there is a constant $c \geq 1$ such that for all $x \in X$ and all positive $r<\frac{1}{4} \operatorname{diam} X$, there exists $E \subset X$ containing $x$ such that $\operatorname{diam} E \leq r$ and $\operatorname{dist}(E, X \backslash E) \geq r / c$. All uniformly disconnected spaces are totally disconnected; on the other hand, $\mathbb{Z}$ is totally disconnected but not uniformly disconnected.

Received by the editors January 24, 2021, and, in revised form, May 17, 2021.

2020 Mathematics Subject Classification. Primary 30C65; Secondary 30L05.

Key words and phrases. Quasisymmetric, bi-Lipschitz, Cantor set, uniformly perfect, uniformly disconnected.

The author was partially supported by the Academy of Finland project 257482 and NSF DMS grant 1952510. 
Based on these scale-invariant notions, the David and Semmes uniformization is as follows.

Theorem 1.1 ([6, Proposition 15.11]). A metric space is quasisymmetrically homeomorphic to $\mathcal{C}$ if and only if it is compact, doubling, uniformly disconnected and uniformly perfect.

Recall that a metric space $X$ is doubling if there exists a constant $C>1$ such that for all $x \in X$ and all $r>0$, the ball $B(x, r)$ can be covered by at most $C$ many balls of radius $r / 2$. Since all Euclidean spaces $\mathbb{R}^{n}$ are doubling, the doubling condition in Theorem 1.1 can be dropped if $X \subset \mathbb{R}^{n}$.

Later, MacManus [15] proved a stronger uniformization result for Cantor sets contained in $\mathbb{R}^{2}$. The improvement here is that the quasisymmetric homeomorphism can be in fact assummed to be defined on the ambient space $\mathbb{R}^{2}$ and not just $\mathcal{C}$.

Theorem 1.2 ([15, Theorem 3]). For a compact set $X \subset \mathbb{R}^{2}$ there exists a quasisymmetric mapping $F: \mathbb{R}^{2} \rightarrow \mathbb{R}^{2}$ with $F(\mathcal{C})=X$ if and only if $X$ is uniformly perfect and uniformly disconnected.

Note that Theorem 1.2 is false in $\mathbb{R}^{3}$ due to the existence of a self-similar wild Cantor set in $\mathbb{R}^{3}$ called Antoine necklace; see [5, pp. 70-75]. By self-similarity, this set is both uniformly perfect and uniformly disconnected, but there exists no homeomorphism of $\mathbb{R}^{3}$ (let alone a quasisymmetric homeomorphism) that maps this set onto $\mathcal{C}$. See also [16, Appendix A] for recent examples in $\mathbb{R}^{4}$.

Additionally, the wildness of $X$ is not the only obstruction in the generalization of MacManus' result. In particular, there exists a compact uniformly perfect and uniformly disconnected set $X \subset \mathbb{R}^{3}$ which is "topologically tame and quasisymmetrically wild", that is, it is ambiently homeomorphic to $\mathcal{C}$ but not ambiently quasisymmetric to $\mathcal{C}$; see [9, Proposition 1.4].

In our main result, we provide a quasisymmetric taming of Cantor sets with bounded geometry. In particular, we show that by increasing the dimension by 1 , MacManus' result generalizes to all dimensions $n \geq 3$.

Theorem A. Let $n \in \mathbb{N}$. For a compact set $X \subset \mathbb{R}^{n}$ there exists a quasisymmetric map $F: \mathbb{R}^{n+1} \rightarrow \mathbb{R}^{n+1}$ with $F(\mathcal{C})=X$ if and only if $X$ is uniformly perfect and uniformly disconnected.

Here and for the rest, given $n \in \mathbb{N}$, we identify $\mathbb{R}^{n}$ with the plane $\mathbb{R}^{n} \times\{0\}=$ $\left\{\left(x_{1}, \ldots, x_{n}, 0\right): x_{i} \in \mathbb{R}\right\} \subset \mathbb{R}^{n+1}$.

One application of Theorem $\mathrm{A}$ is in conformal dynamics. A uniformly quasiregular map (abbv. UQR map) $f: \mathbb{R}^{n} \rightarrow \mathbb{R}^{n}$ is a map for which there exists $K \geq 1$ such that for any $m \in \mathbb{N}$, the $m$-th iterate $f_{m}=f \circ \cdots \circ f$ is in the Sobolev space $W_{\text {loc }}^{1, n}\left(\mathbb{R}^{n}\right)$ and satisfies

$$
\left|f_{m}^{\prime}(x)\right| \leq K J_{f_{m}}, \quad \text { for a.e. } x \in \mathbb{R}^{n} .
$$

Due to the rigidity of conformal maps in dimensions $n \geq 3$, UQR maps play the role of holomorphic maps in the study of conformal dynamics in higher dimensions. A well known problem in conformal dynamics is the characterization of closed sets in $\mathbb{R}^{n}$ that arise as Julia sets of UQR maps. Iwaniecz and Martin [12] showed that the Cantor set $\mathcal{C}$ is a Julia set of a UQR map of $\mathbb{R}^{2}$, and Fletcher and Wu [10] showed that the Antoine necklace is a Julia set of a UQR map of $\mathbb{R}^{3}$. Note that both $\mathcal{C}$ and the Antoine necklace are uniformly perfect and uniformly disconnected because 
they are self-similar. In general, it is unknown whether self-similar Cantor sets in dimensions $n \geq 3$ are always Julia sets of UQR maps. In 9] we apply Theorem A to show that every uniformly perfect and uniformly disconnected subset of $\mathbb{R}^{n}$, $n \geq 3$, is the Julia set of a UQR map of $\mathbb{R}^{n+1}$.

Moreover, Theorem 1.2 and Theorem $\mathrm{A}$ yield the following quasiconformal embedding result for uniformly disconnected sets.

Corollary A. Let $n \geq 2$ be an integer and let $X \subset \mathbb{R}^{n}$ be a bounded uniformly disconnected set. There exists a quasisymmentric homeomorphism of $\mathbb{R}^{N}$ that maps $X$ into $\mathcal{C}$, where $N=2$ if $n=2$, and $N=n+1$ if $n \geq 3$.

Corollary $\mathrm{A}$ has an application in hyperbolic geometry. If $X \subset \mathbb{S}^{2}$ is a Cantor set, then by the Uniformization Theorem, $S:=\mathbb{S}^{2} \backslash X$ is necessarily a hyperbolic Riemann surface. Hence, $S$ has a pants decomposition, that is, $S=\bigcup_{i=1}^{\infty} P_{i}$, where each $P_{i}$ is a topological sphere with three disks removed. The collection of boundary curves of the pairs of pants, called the cuffs of the decomposition, may be enumerated by $\left(\alpha_{j}\right)_{j=1}^{\infty}$. Each $\alpha_{j}$ is a simple closed curve on $S$ and generates a class $\left[\alpha_{j}\right]$ of simple closed curves that are freely homotopic to $\alpha_{j}$. Denote by $\ell_{S}\left[\alpha_{j}\right]$ the infimum of hyperbolic lengths of curves in $\left[\alpha_{j}\right]$. Pommerenke [17] proved that the Cantor set $X$ is uniformly perfect if and only if inf $\ell\left[\alpha_{j}\right]>0$. In a recent paper with Fletcher [8], we apply Corollary A to show that a similar statement holds for uniformly disconnected sets: a Cantor set $X \subset \mathbb{R}^{2}$ is uniformly disconnected if and only if there exists a pair of pants decomposition such that the associated cuffs satisfy $\sup \ell\left[\alpha_{j}\right]<\infty$.

By properties of quasisymmetric homeomorphisms, one direction of Theorem $\mathrm{A}$ is clear. Namely, if there exists a quasisymmetric map $F: \mathbb{R}^{n+1} \rightarrow \mathbb{R}^{n+1}$ with $F(\mathcal{C})=X$, then $X$ is compact, uniformly perfect and uniformly disconnected. For the converse, which is the content of this paper, we use the existence of a quasisymmetric homeomorphism $f: \mathcal{C} \rightarrow X$, and we extend this mapping quasisymmetrically to $\mathbb{R}^{n+1}$. In $\$ 2$ we give some basic definitions, in $\$ 3$ we discuss some bi-Lipschitz Schoenflies theorems in higher dimensions, and in 4 we prove Theorem $\mathrm{A}$ and Corollary A.

\section{Preliminaries}

For $n \in \mathbb{N}$, a point $x \in \mathbb{R}^{n}$, and $r>0$ we denote by $B^{n}(x, r)$ and $\overline{B^{n}}(x, r)$ the open and closed, respectively, ball centered at $x$ and with radius $r$.

A homeomorphism $f: D \rightarrow D^{\prime}$ between two domains in $\mathbb{R}^{n}$ is called $K$-quasiconformal for some $K \geq 1$ if, for all $x \in D, f$ satisfies the distortion inequality

$$
\limsup _{r \rightarrow 0} \frac{\sup _{y \in \partial B^{n}(x, r)}|f(x)-f(y)|}{\inf _{y \in \partial B^{n}(x, r)}|f(x)-f(y)|} \leq K .
$$

A homeomorphism $f:\left(X, d_{X}\right) \rightarrow\left(Y, d_{Y}\right)$ between metric spaces is said to be $\eta$-quasisymmetric if there exists a homeomorphism $\eta:[0, \infty) \rightarrow[0, \infty)$ such that for all $x, a, b \in X$ with $x \neq b$

$$
\frac{d_{Y}(f(x), f(a))}{d_{Y}(f(x), f(b))} \leq \eta\left(\frac{d_{X}(x, a)}{d_{X}(x, b)}\right) .
$$

A quasisymmetric mapping between two domains in $\mathbb{R}^{n}$ is quasiconformal. The converse holds true for the smaller class of uniform domains which contains $\mathbb{R}^{n}$. For a systematic treatment of quasiconformal mappings see 23 . 
It follows easily from the definitions above that quasisymmetric maps preserve the notions of uniform perfectness and uniform disconnectedness quantitatively.

Lemma 2.1 ([14, Theorem 1.3.4]). If $f: X \rightarrow Y$ is $\eta$-quasisymmetric and $X$ is c-uniformly perfect (resp. c-uniformly disconnected), then $Y$ is $c^{\prime}$-uniformly perfect (resp. $c^{\prime}$-uniformly disconnected) with $c^{\prime}$ depending only on $\eta$ and $c$.

A map $f: X \rightarrow Y$ between metric spaces is $L$-bi-Lipschitz for some $L \geq 1$ if

$$
L^{-1} d_{X}(x, y) \leq d_{Y}(f(x), f(y)) \leq L d_{X}(x, y)
$$

for all $x, y \in X$. Note that an $L$-bi-Lipschitz mapping is $L^{2} t$-quasisymmetric.

A weaker notion of bi-Lipschitz mappings is that of bounded length distortion $(B L D)$ mappings. A mapping $f:\left(X, d_{X}\right) \rightarrow\left(Y, d_{Y}\right)$ is $L$-BLD if there exists $L \geq 1$ such that

$$
L^{-1} \ell(\gamma) \leq \ell(f(\gamma)) \leq L \ell(\gamma)
$$

for all paths $\gamma:[0,1] \rightarrow X$. Here and for the rest, $\ell$ denotes the length of a path. Clearly, $L$-bi-Lipschitz mappings are $L$-BLD mappings but BLD mappings need not be bi-Lipschitz even if they are homeomorphisms. However, BLD homeomorphisms between geodesic spaces are bi-Lipschitz.

Lemma 2.2. Let $f: X \rightarrow Y$ be an $L$-BLD homeomorphism between two geodesic metric spaces. Then $f$ is L-bi-Lipschitz.

An embedding $f:\left(X, d_{X}\right) \rightarrow\left(Y, d_{Y}\right)$ is a $(\lambda, L)$-quasisimilarity for some $\lambda>0$ and $L \geq 1$ if

$$
L^{-1} \lambda d_{X}(x, y) \leq d_{Y}(f(x), f(y)) \leq L \lambda d_{X}(x, y) \quad \text { for all } \quad x, y \in X .
$$

Note that $(\lambda, 1)$-quasisimilarities are similarities with scaling factor $\lambda$, while $(1, L)$ quasisimilarities are $L$-bi-Lipschitz, and $(1,1)$-quasisimilarites are isometries.

While similarities preserve relative distances between nondegenerate sets, quasisymmetric maps quasi-preserve relative distances between nondegenerate sets. Specifically, if $f: X \rightarrow Y$ is $\eta$-quasisymmetric and $E, E^{\prime} \subset X$ are nondegenerate closed sets, then

$$
\frac{1}{2} \phi\left(\frac{\operatorname{dist}\left(E, E^{\prime}\right)}{\operatorname{diam} E}\right) \leq \frac{\operatorname{dist}\left(f(E), f\left(E^{\prime}\right)\right)}{\operatorname{diam} f(E)} \leq \eta\left(2 \frac{\operatorname{dist}\left(E, E^{\prime}\right)}{\operatorname{diam} E}\right),
$$

where $\phi(t)=\left(\eta\left(t^{-1}\right)\right)^{-1}$; see for example [22, p. 532]. Moreover, if $f: X \rightarrow Y$ is $\eta$-quasisymmetric and $A \subset B \subset X$ are such that $0<\operatorname{diam} A \leq \operatorname{diam} B<\infty$, then $\operatorname{diam} f(B)$ is finite and

$$
\left(2 \eta\left(\frac{\operatorname{diam} B}{\operatorname{diam} A}\right)\right)^{-1} \leq \frac{\operatorname{diam} f(A)}{\operatorname{diam} f(B)} \leq \eta\left(2 \frac{\operatorname{diam} A}{\operatorname{diam} B}\right) .
$$

For the proof of (2.2) see [11, Proposition 10.8].

\section{Bi-Lipschitz Schoenflies theorems For Multiply CONNECTED DOMAins}

The classical Schönflies theorem states that every embedding of $\mathbb{S}^{1}$ in $\mathbb{R}^{2}$ extends to a homeomorphism of $\mathbb{R}^{2}$. Tukia [19] proved a bi-Lipschitz version of Schönflies theorem.

Theorem 3.1 ([19]). If $f: \mathbb{S}^{1} \rightarrow \mathbb{R}^{2}$ is L-bi-Lipschitz, then $f$ extends in an $L^{\prime}$-biLipschitz way to $\mathbb{R}^{2}$ with $L^{\prime}$ depending only on $L$. 
It is well known that in higher dimensions Theorem 3.1 fails even under strong topological assumptions. In particular, Tukia [19, §15] constructed a bi-Lipschitz embedding of $\mathbb{S}^{2}$ into $\mathbb{R}^{3}$ that can be extended as a homeomorphism of $\mathbb{R}^{3}$ but not as a quasisymmetric (let alone bi-Lipschitz) homeomorphism of $\mathbb{R}^{3}$.

Theorem 3.1 was generalized for annuli in $\mathbb{R}^{2}$ and annuli in higher dimensions by Sullivan [18. Given $L>1$ and integer $n \geq 2$, we say that a domain $D \subset \mathbb{R}^{n}$ is an $L$ bi-Lipschitz ball, if it is the image of the unit ball $\mathbb{B}^{n}$ under a $(\lambda, L)$-quasisimilarity homeomorphism of $\mathbb{R}^{n}$. For the proof of the following theorem see Theorem 3.17 and $\S 5.9$ in $[21$.

Theorem 3.2. Given $n \in\{2,3, \ldots\}, L_{0}, L>1$, and $\delta \in(0,1)$ there exists $L^{\prime}>1$ with the following property. Let $D_{1} \subset D_{2} \subset \mathbb{R}^{n}$ be two $L_{0}$-bi-Lipschitz balls such that

$$
\operatorname{dist}\left(D_{1}, \partial D_{2}\right) \geq \delta \operatorname{diam} D_{2} \quad \text { and } \quad \operatorname{diam} D_{1} \geq \delta \operatorname{diam} D_{2} .
$$

If $f: \partial D_{1} \cup \partial D_{2} \rightarrow \mathbb{R}^{n}$ is an L-bi-Lipschitz embedding that can be extended homeomorphically to $\overline{D_{2}} \backslash D_{1}$ then $f$ extends in an $L^{\prime}$-bi-Lipschitz way to $\overline{D_{2}} \backslash D_{1}$.

In this section, we generalize Theorem 3.2 to multiply connected domains with controlled topology and geometry.

For $d>1, \lambda>1$, and $n \in\{2,3, \ldots\}$ denote by $\mathscr{U}_{n}(\lambda, d)$ the collection of bounded domains $U \subset \mathbb{R}^{n}$ whose boundary components are boundaries of $\lambda$-bi-Lipschitz balls of diameters and mutual distances bounded below by $d^{-1} \operatorname{diam} U$.

Proposition 3.3 is the main result of this section.

Proposition 3.3. Let $U \in \mathscr{U}_{n}(\lambda, d)$ and $f: \partial U \rightarrow \mathbb{R}^{n}$ be an L-bi-Lipschitz map that extends homeomorphically to $\bar{U}$. Then $f$ extends in an $L^{\prime}$-bi-Lipschitz way to $\bar{U}$ with $L^{\prime}$ depending only on $L, \lambda, d$, and $n$.

We start with the simple observation that every domain in $\mathscr{U}_{n}(\lambda, d)$ has a finite number of boundary components.

Lemma 3.4. Every domain $U \in \mathscr{U}_{n}(\lambda, d)$ contains at most $N$ boundary components with $N$ depending only on $n, d$.

Proof. Let $D_{1}, \ldots, D_{m}$ be some bounded components of $\mathbb{R}^{n} \backslash \bar{U}$. For each $i \in$ $\{1, \ldots, m\}$, choose $x_{i} \in D_{i}$. Then, for each distinct $i, j$, we have $x_{i} \in \overline{B^{n}}\left(x_{1}, \operatorname{diam} U\right)$ and $\left|x_{i}-x_{j}\right| \geq d^{-1} \operatorname{diam} U$. By the doubling property of $\mathbb{R}^{n}$, we have that $m \leq$ $N_{0} d^{-n}$ for some universal $N_{0}$.

Below, for a domain $U \in \mathscr{U}_{n}(\lambda, d)$, we write $U=U\left(D_{0} ; D_{1}, \ldots, D_{m}\right)$ if $D_{0}, \ldots, D_{m}$ are $\lambda$-bi-Lipschitz balls with

(1) $U=D_{0} \backslash \bigcup_{i=1}^{m} \overline{D_{i}}$;

(2) $\overline{D_{1}}, \ldots, \overline{D_{m}}$ are contained in $D_{0}$ and are mutually disjoint;

(3) for all $i \in\{1, \ldots, m\}$ we have $\operatorname{diam} D_{i} \geq d^{-1} \operatorname{diam} D_{0}$;

(4) for all distinct $i, j \in\{0, \ldots, m\}$ we have $\operatorname{dist}\left(\partial D_{i}, \partial D_{j}\right) \geq d^{-1} \operatorname{diam} D_{0}$.

For the rest of $\$ 3$ we denote by $\mathcal{S}_{0}$ the open $n$-cube $(-1,1)^{n}$ in $\mathbb{R}^{n}$. For each $m \in \mathbb{N}$ and $k \in\{1, \ldots, m\}$ define the open cubes

$$
\mathcal{S}_{m, k}:=\left(\frac{4 k-2 m-3}{2 m+1}, \frac{4 k-2 m-1}{2 m+1}\right) \times\left(\frac{-1}{2 m+1}, \frac{1}{2 m+1}\right)^{n-1} \subset \mathbb{R}^{n}
$$



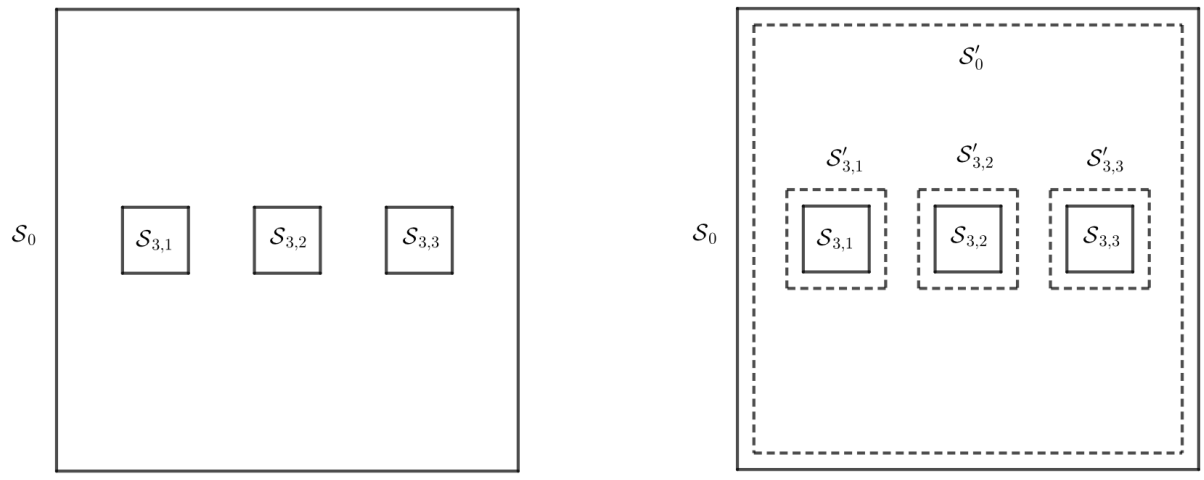

FiguRE 1. The profile of cubes $\mathcal{S}_{0}, \mathcal{S}_{m, k}, \mathcal{S}_{0}^{\prime}$, and $\mathcal{S}_{m, k}^{\prime}$ in the first two dimensions in the case $m=3$

and the domains

$$
\mathcal{U}_{0}:=\mathcal{S}_{0}, \quad \mathcal{U}_{m}:=\mathcal{S}_{0} \backslash \bigcup_{k=1}^{m} \overline{\mathcal{S}_{m, k}}, \quad \text { for } m \in \mathbb{N} .
$$

In Lemma 3.5, we show that every domain $U \in \mathscr{U}_{n}(\lambda, d)$ is quasisimilar to $\mathcal{U}_{m}$ for some $m \in \mathbb{N}$. This allows us to reduce the proof of Proposition 3.3 to the case $U=\mathcal{U}_{m}$.

Lemma 3.5. For $n \in\{2,3, \ldots\}, \lambda>1$, and $d>0$ there exists $L \geq 1$ with the following property. If $U=U\left(D_{0} ; D_{1}, \ldots, D_{m}\right)$ is in $\mathscr{U}_{n}(\lambda, d)$ and has diameter equal to 1 , then there exists an L-bi-Lipschitz homeomorphism $f: \bar{U} \rightarrow \overline{\mathcal{U}_{m}}$ with $f\left(\partial D_{0}\right)=\partial \mathcal{S}_{0}$ and $f\left(\partial D_{i}\right)=\partial \mathcal{S}_{m, i}$ for $i=1, \ldots, m$.

Note that the order of domains $D_{i}, i \in\{1, \ldots, m\}$ is arbitrary. Therefore, Lemma 3.5 implies that we can bi-Lipschitz map $U$ onto $\mathcal{U}_{m}$ and match the cubes $\partial \mathcal{S}_{m, i}$ with the inner boundary components of of $U$, in any order.

For the proof of Lemma 3.5. given $k \in \mathbb{N}$ we say that a point $x \in \mathbb{R}^{n}$ is a $k$-dyadic point if there exists $\left(i_{1}, \ldots, i_{n}\right) \in \mathbb{Z}^{n}$ such that $x=\left(i_{1} 2^{-k}, \ldots, i_{n} 2^{-k}\right)$.

Remark 3.6. For any $k \in \mathbb{N}$ and any $x \in \mathbb{R}^{n}$, there exists a $k$-dyadic point $x^{\prime} \in \mathbb{R}^{n}$ such that $\left|x-x^{\prime}\right|<2^{-k} \sqrt{n}$.

To prove Lemma 3.5 we require the following result.

Lemma 3.7. Let $\left\{p_{1}, \ldots, p_{m}\right\}$ and $\left\{q_{1}, \ldots, q_{m}\right\}$ be two families of distinct $k$-dyadic points in $\mathcal{S}_{0}$. Given $r=\frac{1}{10} 2^{-k}$, there exists $L>1$ depending only on $n$ and $k$, and there exists an L-bi-Lipschitz homeomorphism

$$
f: \overline{\mathcal{S}_{0}} \backslash \bigcup_{i=1}^{m} B^{n}\left(p_{i}, r\right) \rightarrow \overline{\mathcal{S}_{0}} \backslash \bigcup_{i=1}^{m} B^{n}\left(q_{i}, r\right)
$$

such that for all $i, f$ maps $\partial B^{n}\left(p_{i}, r\right)$ onto $\partial B^{n}\left(q_{i}, r\right)$.

Proof. Let $l_{1}$ denote the line segment $\left[p_{1}, q_{1}\right]$. Then $\operatorname{dist}\left(l_{1}, \partial \mathcal{S}_{0}\right) \geq 2^{-k}$. We modify $l_{1}$ on its intersections with balls $B^{n}\left(p_{i}, 5 r\right)$ for $i \neq 1$ replacing $l_{1} \cap B^{n}\left(p_{i}, 5 r\right)$ (if 
nonempty) by an arc on $\partial B^{n}\left(p_{i}, 5 r\right)$ of minimal length. We denote the new curve by $\gamma_{1}$. Since we have chosen $r$ small enough, it follows that the set

$$
D_{1}=\bigcup_{x \in \gamma_{1}} B^{n}(x, 2 r)
$$

is a $\lambda_{1}$-bi-Lipschitz ball. In fact, since there are a finite number of arrangement for the points $\left\{p_{1}, \ldots, p_{m}, q_{1}\right\}$ in $\mathcal{S}_{0}$ (depending only on $k$ and $n$ ), we have that $\lambda_{1}$ depends only on $n$ and $k$. Moreover, $D_{1}$ contains $B^{n}\left(p_{1}, r\right)$ and $B^{n}\left(q_{1}, r\right)$ and does not intersect any of the balls $B^{n}\left(p_{i}, r\right)$ for $i \neq 1$. Applying Theorem 3.2 on $\overline{D_{1}} \backslash B^{n}\left(p_{1}, r\right)$, there exists $L_{1}>1$ depending only on $k, n$ and an $L_{1}$-bi-Lipschitz map

$$
f_{1}: \overline{\mathcal{S}_{0}} \backslash \bigcup_{i=1}^{m} B^{n}\left(p_{i}, r\right) \rightarrow \overline{\mathcal{S}_{0}} \backslash\left(B^{n}\left(q_{1}, r\right) \cup \bigcup_{i=2}^{m} B^{n}\left(p_{i}, r\right)\right)
$$

such that $f_{1}$ is identity on $\overline{\mathcal{S}_{0}} \backslash D_{1}$ and maps $\partial B\left(p_{1}, r\right)$ onto $\partial B\left(q_{1}, r\right)$.

Working as above, for each $j \in\{2, \ldots, m\}$, there exists an $L_{j}$-bi-Lipschitz map

$$
f_{j}: \overline{\mathcal{S}_{0}} \backslash\left(\bigcup_{i=j}^{m} B^{n}\left(p_{i}, r\right) \cup \bigcup_{i=1}^{j-1} B^{n}\left(q_{i}, r\right)\right) \rightarrow \overline{\mathcal{S}_{0}} \backslash\left(\bigcup_{i=1}^{j} B^{n}\left(q_{i}, r\right) \cup \bigcup_{i=j+1}^{m} B^{n}\left(p_{i}, r\right)\right)
$$

such that $L_{j}$ depends only on $n, k$, the map $f_{j}$ is identity on

$$
\bigcup_{i=j+1}^{m} B^{n}\left(p_{i}, r\right) \cup \bigcup_{i=1}^{j-1} B^{n}\left(q_{i}, r\right)
$$

and $f_{j}$ maps $\partial B\left(p_{j}, r\right)$ onto $\partial B\left(q_{j}, r\right)$. Here we use the convention $\bigcup_{i=j+1}^{m} B^{n}\left(p_{i}, r\right)$ $=\emptyset$ if $j=m$. Define now

$$
f=f_{m} \circ \cdots \circ f_{1}: \overline{\mathcal{S}_{0}} \backslash \bigcup_{i=1}^{m} B^{n}\left(q_{i}, r\right) \rightarrow \overline{\mathcal{S}_{0}} \backslash \bigcup_{i=1}^{m} B^{n}\left(p_{i}, r\right) .
$$

Note that $f$ maps each $\partial B^{n}\left(p_{i}, r\right)$ onto $\partial B^{n}\left(q_{i}, r\right)$. Since $m \leq\left(2^{k+1}+1\right)^{n}$, the bi-Lipschitz constant of $f$ depends only on $n, k$.

We can now show Lemma 3.5 .

Proof of Lemma 3.5. By Lemma 3.4, $m \leq N$ for some $N$ depending only on $d$ and $n$. Applying a $\lambda_{0}$-bi-Lipschitz homeomorphism of $\mathbb{R}^{n}$ for some $\lambda_{0}>1$ depending only on $\lambda$ and $n$, we may assume that $D_{0}=\mathcal{S}_{0}$.

Let us outline the proof. In the first step we shrink the domains $D_{i}$ isotopically to small balls with dyadic centers. In the second step we apply Lemma 3.7 to move these balls to some arranged positions and in the third step we move the balls from these positions to balls inside the cubes $\mathcal{S}_{m, k}$. In the fourth and final step we inflate these balls isotopically to cubes $\mathcal{S}_{m, i}$.

To this end, we define three collections $\left\{x_{1}, \ldots, x_{m}\right\},\left\{y_{1}, \ldots, y_{m}\right\},\left\{z_{1}, \ldots, z_{m}\right\}$ of dyadic points. Let $k \in \mathbb{N}$ be the smallest integer such that

$$
2^{-k} \leq\left(2 \lambda^{2} \sqrt{n}(2 m+1) d\right)^{-1} .
$$

First, there exists $x_{0} \in U$ such that

$$
\operatorname{dist}\left(x_{0}, \partial U\right) \geq(2 d)^{-1} \operatorname{diam} U=(2 d)^{-1} \operatorname{diam} \mathcal{S}_{0}=d^{-1} \sqrt{n} .
$$


By Remark 3.6, there exists a $k$-dyadic point $x_{0}^{\prime}$ such that $\left|x_{0}^{\prime}-x_{0}\right|<2^{-k} \sqrt{n}$. By the choice of $k$, the $k$-dyadic points

$$
x_{i}=x_{0}^{\prime}+2^{-k}(i-1)(1,0, \ldots, 0), \quad i=1, \ldots, m
$$

satisfy

$$
\begin{aligned}
\operatorname{dist}\left(x_{i}, \partial U\right) & \geq \operatorname{dist}\left(x_{0}, \partial U\right)-\left|x_{0}-x_{0}^{\prime}\right|-\left|x_{0}^{\prime}-x_{i}\right| \\
& \geq d^{-1} \sqrt{n}-2^{-k} \sqrt{n}-(m-1) 2^{-k} \\
& \geq \frac{1}{2} \sqrt{n} d^{-1} .
\end{aligned}
$$

Second, since each domain $D_{i}$ is a $\lambda$-bi-Lipschitz ball of diameter at least $d^{-1} \sqrt{n}$, there exist for each $i$ a point $y_{i}^{\prime} \in D_{i}$ such that $\operatorname{dist}\left(y_{i}^{\prime}, \partial D_{i}\right) \geq \lambda^{-2} d^{-1} \sqrt{n}$. By Remark 3.6. there exists a $k$-dyadic point $y_{i}$ such that $\left|y_{i}-y_{i}^{\prime}\right|<2^{-k} \sqrt{n}$. By the choice of $k$, we have that $y_{i} \in D_{i}$ and

$$
\operatorname{dist}\left(y_{i}, \partial D_{i}\right) \geq \operatorname{dist}\left(y_{i}^{\prime}, \partial D_{i}\right)-\left|y_{i}-y_{i}^{\prime}\right| \geq \frac{1}{2} \lambda^{-2} d^{-1} \sqrt{n} .
$$

Third, for each $i$ let $z_{i}^{\prime}$ be the center of the open cube $\mathcal{S}_{m, i}$. By Remark 3.6, there exists a $k$-dyadic point $z_{i}$ such that $\left|z_{i}-z_{i}^{\prime}\right|<2^{-k} \sqrt{n}$. By the choice of $k$,

$$
\operatorname{dist}\left(z_{i}, \partial \mathcal{S}_{m, i}\right) \geq(2 m+1)^{-1}-2^{-k} \sqrt{n} \geq \frac{1}{2}(2 m+1)^{-1} .
$$

We now construct four bi-Lipschitz homeomorphisms. Let

$$
r=\frac{1}{10} \min \left\{2^{-k}, \frac{1}{2}(2 m+1)^{-1}, \frac{1}{2} \lambda^{-2} d^{-1} \sqrt{n}\right\}=\frac{1}{10} 2^{-k} .
$$

First, for each $i$, let $B^{n}\left(a_{i}, R_{i}\right)$ be a ball and let $g_{i}$ be a $\lambda$-bi-Lipschitz homeomorphism of $\mathbb{R}^{n}$ mapping $B^{n}\left(a_{i}, R_{i}\right)$ onto $D_{i}$. Note that $\frac{2 \sqrt{n}}{\lambda d} \leq R_{i} \leq 2 \lambda \sqrt{n}$. Let $D_{i}^{\prime}=g_{i}\left(B\left(a_{i},\left(1+\left(2 \lambda^{2} d\right)^{-1}\right)\right)\right.$. Then, $D_{i}^{\prime}$ is a $\lambda$-bi-Lipschitz ball containing $D_{i}$ such that the Hausdorff distance

$$
\frac{1}{2}(\lambda d)^{-2} \sqrt{n} \leq \operatorname{dist}_{H}\left(\partial D_{i}, \partial D_{i}^{\prime}\right) \leq \frac{1}{2} d^{-1} \sqrt{n} .
$$

Therefore, for each $i$ we have that $\partial D_{i}^{\prime} \subset U$ and for all distinct $i, j$ we have $\operatorname{dist}\left(\partial D_{i}^{\prime}, \partial D_{j}^{\prime}\right) \geq d^{-1} \sqrt{n}$. Applying Theorem 3.2 on each annulus $\overline{D_{i}} \backslash D_{i}^{\prime}$ we obtain an $L_{1}$-bi-Lipschitz map

$$
F_{1}: \bar{U} \rightarrow \overline{\mathcal{S}_{0}} \backslash \bigcup_{i=1}^{m} B^{n}\left(y_{i}, r\right)
$$

so that $F_{1}$ is the identity on $\overline{\mathcal{S}_{0}} \backslash \bigcup_{i=1}^{m} D_{i}^{\prime}$ and maps each $\partial D_{i}$ onto $\partial B^{n}\left(y_{i}, \frac{3}{2} r\right)$, with $L_{1}$ depending only on $n, \lambda, d$.

Second, for each $i=1, \ldots, m$ following the same ideas as above, we can construct an $L_{2}$-bi-Lipschitz map

$$
F_{2}: \overline{\mathcal{U}_{m}} \rightarrow \overline{\mathcal{S}_{0}} \backslash \bigcup_{i=1}^{m} B^{n}\left(z_{i}, r\right)
$$

with $F_{2}\left(\partial \mathcal{S}_{m, i}\right)=\partial B\left(z_{i}, r\right)$, and $L_{2}$ depending only on $n$ and $d$.

Third, by Lemma 3.7, there exists an $L_{3}$-bi-Lipschitz homeomorphism

$$
F_{3}: \overline{\mathcal{S}_{0}} \backslash \bigcup_{i=1}^{m} B^{n}\left(y_{i}, r\right) \rightarrow \overline{\mathcal{S}_{0}} \backslash \bigcup_{i=1}^{m} B^{n}\left(x_{i}, r\right)
$$

with $F_{3}\left(\partial B^{n}\left(y_{i}, r\right)\right)=\partial B^{n}\left(x_{i}, r\right)$, and with $L_{3}>1$ depending only on $n, k$, hence only on $n, \lambda, d$. 
Fourth, by Lemma 3.7, there exists an $L_{4}$-bi-Lipschitz homeomorphism

$$
F_{4}: \overline{\mathcal{S}_{0}} \backslash \bigcup_{i=1}^{m} B^{n}\left(z_{i}, r\right) \rightarrow \overline{\mathcal{S}_{0}} \backslash \bigcup_{i=1}^{m} B^{n}\left(x_{i}, r\right)
$$

with $F_{4}\left(\partial B^{n}\left(z_{i}, r\right)\right)=\partial B^{n}\left(x_{i}, r\right)$ and $L_{4}$ depending only on $n, \lambda, d$.

The map $F_{2}^{-1} \circ F_{4}^{-1} \circ F_{3} \circ F_{1}: \bar{U} \rightarrow \overline{\mathcal{U}_{m}}$ is an $L$-bi-Lipschitz homeomorphism such that $F\left(\partial D_{i}\right)=\partial \mathcal{S}_{m, i}$ for all $i \in\{1, \ldots, m\}$, and with $L$ depending only on $n$, $\lambda$, and $d$.

We are now ready to prove Proposition 3.3 ,

Proof of Proposition 3.3 . Proposition 3.3 is trivial if $\partial U$ has only one component. For the rest, we assume that $\partial U$ has at least two components.

Since the embedding $f$ can be extended homeomorphically to $\bar{U}$, there exists a domain $U^{\prime} \subset \mathbb{R}^{n}$ such that $\partial U^{\prime}=f(\partial U)$ and $f$ can be extended to a homeomorphism of $\bar{U}$ onto $\overline{U^{\prime}}$. Applying two similarities with comparable scaling factors, we may assume that $\operatorname{diam} U=\operatorname{diam} U^{\prime}=1$. Since $f$ is $L$-bi-Lipschitz, we have that $U^{\prime} \in \mathscr{U}_{n}\left(\lambda^{\prime}, d^{\prime}\right)$ for some $\lambda^{\prime}, d^{\prime}>1$ depending only on $n, \lambda, d$. By Lemma 3.5, applying two $L_{0}$-bi-Lipschitz maps, with $L_{0}$ depending only on $n, \lambda, d$, we may assume that $U=U^{\prime}=\mathcal{U}_{m}$, that $f$ maps $\partial \mathcal{S}_{0}$ onto $\partial \mathcal{S}_{0}$, and for each $k \in\{1, \ldots, m\}, f$ maps $\partial \mathcal{S}_{m, k}$ onto $\partial \mathcal{S}_{m, k}$.

Define the open cube

$$
\mathcal{S}_{0}^{\prime}:=\left(\frac{1 / 2}{2 m+1}-1,1-\frac{1 / 2}{2 m+1}\right)^{n}
$$

and for each $k=1, \ldots, m$ define the open cube

$$
\mathcal{S}_{m, k}^{\prime}:=\left(\frac{4 k-2 m-7 / 2}{2 m+1}, \frac{4 k-2 m-1 / 2}{2 m+1}\right) \times\left(-\frac{3 / 2}{2 m+1}, \frac{3 / 2}{2 m+1}\right)^{n-1}
$$

so that $\mathcal{S}_{m, k} \subset \mathcal{S}_{m, k}^{\prime} \subset \mathcal{S}_{0}^{\prime} \subset \mathcal{S}_{0}$ for each $k=1, \ldots, m$; see Figure 1. Extend $f$ to $\partial \mathcal{S}_{0}^{\prime}$ and to each $\mathcal{S}_{m, k}^{\prime}$ with identity and note that the new embedding, which we still denote by $f$, is $L_{1}$-bi-Lipschitz with $L_{1}$ depending only on $L$ and $m$, hence only on $L, n$, and $d$.

Applying Theorem 3.2 on each $\overline{\mathcal{S}_{k, m}^{\prime}} \backslash \mathcal{S}_{k, m}$ we obtain $L_{1}^{\prime}$-bi-Lipschitz extensions $g_{k}$ on $\overline{\mathcal{S}_{k, m}^{\prime}} \backslash \mathcal{S}_{k, m}$ with $L_{1}^{\prime}$ depending only on $n, d$ and $L$. Similarly, we obtain an $L_{1}^{\prime \prime}$-bi-Lipschitz extension $g_{0}$ on $\mathcal{S}_{0} \backslash \mathcal{S}_{0}^{\prime}$. The map

$$
f: \overline{\mathcal{U}_{m}} \rightarrow \overline{\mathcal{U}_{m}} \quad \text { with } \quad f(x)= \begin{cases}g_{0}(x), & \text { if } x \in \overline{\mathcal{S}_{0}} \backslash \mathcal{S}_{0}^{\prime} \\ x, & \text { if } x \in \overline{\mathcal{S}_{0}^{\prime} \backslash \bigcup_{k} \mathcal{S}_{m, k}^{\prime}} \\ g_{k}(x), & \text { if } x \in \overline{\mathcal{S}_{m, k}^{\prime} \backslash \mathcal{S}_{m, k}}\end{cases}
$$

is $L^{\prime}$-BLD for some $L^{\prime}$ depending only on $n, L$ and $d$. By Lemma 2.2, $f$ is $L^{\prime}$-biLipschitz.

\section{Proof of Theorem $\mathrm{A}$ and Corollary $\mathrm{A}$}

Here we prove the following quantitative version of Theorem A The proof of Corollary $\mathrm{A}$ is given in 4.1 . 
Theorem 4.1. Let $n \in \mathbb{N}$ and let $X \subset \mathbb{R}^{n}$ be a compact c-uniformly perfect and c-uniformly disconnected set. There exists an $\eta^{\prime}$-quasisymmetric homeomorphism $F: \mathbb{R}^{n+1} \rightarrow \mathbb{R}^{n+1}$ with $F(\mathcal{C})=X$, and with $\eta^{\prime}$ depending only on $n$ and $c$.

We define $\mathcal{W}$ to be the set of finite words formed from the letters $\{1,2\}$, including the empty word $\varepsilon$. Define $\mathcal{W}^{N}$ to be the set of words in $\mathcal{W}$ whose length is exactly $N$. Given $w \in \mathcal{W}$, we denote by $|w|$ the number of letters that $w$ has, with $|\varepsilon|=0$.

Let $I_{\varepsilon}=[0,1]$ and given $I_{w}=[a, b]$ let $I_{w 1}=\left[a, a+\frac{1}{3}(b-a)\right], I_{w 2}=\left[b-\frac{1}{3}(b-a), b\right]$. For each $w \in \mathcal{W}$, let $\mathcal{C}_{w}=I_{w} \cap \mathcal{C}$.

Lemma 4.2. Let $X$ be a metric space and let $f: \mathcal{C} \rightarrow X$ be an $\eta$-quasisymmetric homeomorphism. For each $D>1$, there exists $k \in \mathbb{N}$ depending only on $\eta$ and $D$ with the following property. For any integer $m \geq \log k / \log 2$, there exists a partition $\mathscr{E}_{1}, \ldots, \mathscr{E}_{k}$ of $\mathcal{W}^{m}$ such that for any $i \in\{1, \ldots, k\}$ and any distinct $w, w^{\prime} \in \mathscr{E}_{i}$,

$$
\operatorname{dist}\left(f\left(\mathcal{C}_{w}\right), f\left(\mathcal{C}_{w^{\prime}}\right)\right) \geq D \max \left\{\operatorname{diam} f\left(\mathcal{C}_{w}\right), \operatorname{diam} f\left(\mathcal{C}_{w^{\prime}}\right)\right\} .
$$

Proof. Set $d=\left(\eta^{-1}\left((2 D)^{-1}\right)\right)^{-1}$. We show that the lemma holds for $k$ being the integer part of $2 d^{\log 2 / \log 3}+1$. Fix an integer $m \geq \log k / \log 2$. Let $l$ be the integer part of $\log d / \log 3+1$. By definition, $k \geq 2^{l}$. For distinct $u, u^{\prime} \in \mathcal{W}^{m-l}$ we have

$$
\operatorname{dist}\left(\mathcal{C}_{u}, \mathcal{C}_{u^{\prime}}\right) \geq 3^{-(m-l)} \geq d 3^{-m} .
$$

Therefore, for all $w \in \mathcal{W}^{m}$, there exist at most $2^{l}$ (hence at most $k$ ) words $w^{\prime} \in \mathcal{W}^{m}$ such that $\operatorname{dist}\left(\mathcal{C}_{w}, \mathcal{C}_{w^{\prime}}\right) \geq d 3^{-m}$.

Let now $\left\{w_{1}, \ldots, w_{2^{m}}\right\}$ be an enumeration of $\mathcal{W}^{m}$ such that for all $1 \leq i<j \leq$ $2^{m}$, the set $\mathcal{C}_{w_{i}}$ lies to the left of the set $\mathcal{C}_{w_{j}}$. For each $i=1, \ldots, k$ define $A_{i}$ to be the integers in $\left\{1, \ldots, 2^{m}\right\}$ that are of the form $i+r k$ with $r \in \mathbb{N} \cup\{0\}$ and set $\mathscr{E}_{i}=\left\{w_{j}: j \in A_{i}\right\}$. It is now straightforward to see that the sets $\mathscr{E}_{i}$ form a partition of $\mathcal{W}^{m}$ and that for all $i \in\{1, \ldots, k\}$ and all distinct $w, w^{\prime} \in \mathcal{E}_{i}$,

$$
\operatorname{dist}\left(\mathcal{C}_{w}, \mathcal{C}_{w^{\prime}}\right) \geq d 3^{-m}=d \max \left\{\operatorname{diam} \mathcal{C}_{w}, \operatorname{diam} \mathcal{C}_{w^{\prime}}\right\} .
$$

Now inequality (4.1) follows by quasisymmetry of $f$ and (2.1).

We are now ready to prove Theorem 4.1 Let us first give an informal outline of the proof. The proof consists of two steps. The first step is the construction of a bi-Lipschitz mapping $\Phi: \mathbb{R}^{n+1} \rightarrow \mathbb{R}^{n+1}$ that unlinks $X$. Roughly speaking, we apply Lemma 4.2 to break $X$ into several pieces which we lift in specific heights with those being close lifted to different heights while those being far lifted to the same height. Then, we repeat the same process to each of these pieces breaking them into further smaller pieces and lifting them to specific heights. The heights will be of the form of Lipschitz functions $\phi_{i}$ that we define during the proof.

The subsets and heights from the first step are chosen carefully to make sure that the lifted subsets are contained in disjoint cubes. In the second step, we apply Proposition 3.3 to construct a quasiconformal mapping $G: \mathbb{R}^{n+1} \rightarrow \mathbb{R}^{n+1}$ that maps the unlinked image $\Phi(X)$ onto $\mathcal{C}$. The composition $G \circ \Phi$ is the desired map $F$ of Theorem $\mathrm{A}$.

Proof of Theorem 4.1. Let $X$ be a compact, $c$-uniformly perfect and $c$-uniformly disconnected subset of $\mathbb{R}^{n}$. By Theorem 1.1, there exists an $\eta$-quasisymmetric homeomorphism $f: \mathcal{C} \rightarrow X$ with $\eta$ depending only on $n$ and $c$. Without loss of generality, we assume that $\operatorname{diam} X=1$. For the rest of the proof we write 
$X_{w}=f\left(\mathcal{C}_{w}\right)$. Let $k$ be the number obtained by Lemma 4.2 for $D:=1+8 \sqrt{n+1}$ and for $\eta$. Let also $N$ be the smallest positive integer such that

$$
3^{-N} \leq \frac{1}{2} \eta^{-1}\left((20 k \sqrt{n})^{-1}\right) \quad \text { and } \quad N \geq \log k / \log 2 .
$$

By (2.1) and (2.2), for any two distinct $w, w^{\prime} \in \mathcal{W}$ with $|w|=\left|w^{\prime}\right|$, and any $u \in \mathcal{W}^{N}$,

$$
\begin{aligned}
& \left(2 \eta\left(3^{N}\right)\right)^{-1} \operatorname{diam} X_{w} \leq \operatorname{diam} X_{w u} \leq \eta\left(2 \cdot 3^{-N}\right) \operatorname{diam} X_{w}, \\
& \operatorname{dist}\left(X_{w}, X_{w^{\prime}}\right) \geq(2 \eta(1))^{-1} \max \left\{\operatorname{diam} X_{w}, \operatorname{diam} X_{w^{\prime}}\right\} .
\end{aligned}
$$

Let $\mathscr{E}_{1}, \ldots, \mathscr{E}_{k} \varepsilon$ be the sets of Lemma 4.2 corresponding to $f, D$, and $m=N$. Define $\phi_{1}: X \rightarrow \mathbb{R}$ by

$$
\left.\phi_{1}\right|_{X_{w}}(x)=(4 k)^{-1}(i-1), \quad \text { for } w \in \mathscr{E}_{i}^{\varepsilon} \text { and } i \in\{1, \ldots, k\} .
$$

Inductively, suppose that for some $j \in \mathbb{N}$ we have defined $\phi_{j}: X \rightarrow \mathbb{R}$ such that $\left.\phi_{j}\right|_{X_{w}}$ is constant whenever $w \in \mathcal{W}^{j N}$. For each $w \in \mathcal{W}^{j N}$, let $\zeta_{w}: \mathcal{C} \rightarrow \mathcal{C}_{w}$ be a similarity and note that $\left.f\right|_{\mathcal{C}_{w}} \circ \zeta_{w}: \mathcal{C} \rightarrow X_{w}$ is $\eta$-quasisymmetric. Let $\mathscr{E}_{1} w, \ldots, \mathscr{E}_{k} w$ be the sets of $\mathcal{W}^{N}$ from Lemma 4.2 applied to $\left.f\right|_{\mathcal{C}_{w}} \circ \zeta_{w}, D$, and $m=N$. Define $\phi_{j+1}: X \rightarrow \mathbb{R}$ such that

$$
\left.\phi_{j+1}\right|_{X_{w u}}(x)=\left.\phi_{j}\right|_{X_{w}}(x)+(4 k)^{-1}(i-1) \operatorname{diam} X_{w},
$$

where $w \in \mathcal{W}^{j N}, u \in \mathscr{E}_{i}^{w}$ and $i \in\{1, \ldots, k\}$.

By (4.2), for positive integers $j<j^{\prime}$ we have

$$
\begin{aligned}
\left\|\phi_{j}-\phi_{j^{\prime}}\right\|_{\infty} \leq \sum_{l=j}^{j^{\prime}-1}\left\|\phi_{l}-\phi_{l+1}\right\|_{\infty} & \leq \sum_{l=j}^{j^{\prime}-1}(4 k)^{-1}(k-1) \max _{w \in \mathcal{W}^{l N}} \operatorname{diam} X_{w} \\
& \leq \sum_{l=j}^{j^{\prime}-1} \frac{1}{4}\left(\eta\left(2 \cdot 3^{-N}\right)\right)^{l} \\
& \leq \frac{\left(\eta\left(2 \cdot 3^{-N}\right)\right)^{j}}{4-4 \eta\left(2 \cdot 3^{-N}\right)}
\end{aligned}
$$

which, by choice of $N$, goes to 0 as $j \rightarrow \infty$. Therefore, the mappings $\phi_{j}$ converge uniformly to a mapping $\phi: X \rightarrow \mathbb{R}$.

We claim that $\phi$ is Lipschitz with the Lipschitz constant depending only on $n$ and $c$. To see that, let $x, y \in X$ and let $j \in \mathbb{N}$ be the unique integer such that there exists $w \in \mathcal{W}^{j N}$ and there exist distinct $u, u^{\prime} \in \mathcal{W}^{N}$ with $x \in X_{w u}$ and $y \in X_{w u^{\prime}}$. On the one hand, by (4.2), (4.3),

$$
|x-y| \geq \operatorname{dist}\left(X_{w u}, X_{w u^{\prime}}\right) \geq \frac{1}{2 \eta(1)} \min _{v \in \mathcal{W}^{N}} \operatorname{diam} X_{w v} \geq \frac{\operatorname{diam} X_{w}}{4 \eta(1) \eta\left(3^{N}\right)} .
$$

On the other hand, by (4.2) and the fact that $\phi_{j}(x)=\phi_{j}(y)$,

$$
\begin{aligned}
|\phi(x)-\phi(y)| & \leq\left|\phi(x)-\phi_{j}(x)\right|+\left|\phi_{j}(x)-\phi_{j}(y)\right|+\left|\phi(y)-\phi_{j}(y)\right| \\
& \leq 2(4 k)^{-1}(k-1) \operatorname{diam} X_{w} \sum_{l=0}^{\infty}\left(\eta\left(2 \cdot 3^{-N}\right)\right)^{l} \\
& \leq \frac{1}{2-2 \eta\left(2 \cdot 3^{-N}\right)} \operatorname{diam} X_{w} .
\end{aligned}
$$


Therefore,

$$
|\phi(x)-\phi(y)| \leq \frac{2 \eta(1) \eta\left(3^{N}\right)}{1-\eta\left(2 \cdot 3^{-N}\right)}|x-y|
$$

and the claim follows.

Fix $x_{0} \in X$, set $B_{0}=B^{n}\left(x_{0}, 5 \operatorname{diam} X\right)$ and set $\left.\phi\right|_{\mathbb{R}^{n} \backslash B_{0}} \equiv 0$. Then, the map

$$
\phi:\left(\mathbb{R}^{n} \backslash B_{0}\right) \cup X \rightarrow \mathbb{R}
$$

is $L$-Lipschitz for some $L$ depending only on $n, c$ and, by the Kirszbraun Theorem [7. 2.10.43], there exists an $L$-Lipschitz extension of $\phi$ to $\mathbb{R}^{n}$ which we also denote by $\phi$. Then, the mapping

$$
\Phi: \mathbb{R}^{n+1} \rightarrow \mathbb{R}^{n+1}, \quad \text { defined by } \Phi(x, z)=(x, \phi(x)+z)
$$

is $L^{\prime}$-bi-Lipschitz with $L^{\prime}=2(L+2)$ [24, Lemma 5.3.2].

For each $j=0,1, \ldots$ and each $w \in \mathcal{W}^{j N}$ fix $x_{w} \in X_{w}$ and set

$$
\mathrm{K}_{w}:=x_{w}+\left[-2 \operatorname{diam} X_{w}, 2 \operatorname{diam} X_{w}\right]^{n}=\left\{x_{w}+\left(2 \operatorname{diam} X_{w}\right) y: y \in[-1,1]^{n}\right\} .
$$

We claim that if $w \in \mathcal{W}^{j N}$ and $u \in \mathcal{W}^{N}$, then

$$
\mathrm{K}_{w u} \subset \mathrm{K}_{w} \quad \text { and } \quad \operatorname{dist}\left(\mathrm{K}_{w u}, \partial \mathrm{K}_{w}\right) \geq \frac{1}{2} \operatorname{diam} X_{w} .
$$

Indeed, if $x \in \mathrm{K}_{w u}$, then by (4.2) and the choice of $N$,

$$
\begin{aligned}
\left|x-x_{w}\right| \leq\left|x-x_{w u}\right|+\left|x_{w u}-x_{w}\right| & \leq 2 \sqrt{n} \operatorname{diam} X_{w u}+\operatorname{diam} X_{w} \\
& \leq\left(1+2 \sqrt{n} \eta\left(2 \cdot 3^{-N}\right)\right) \operatorname{diam} X_{w} \\
& <\frac{3}{2} \operatorname{diam} X_{w}
\end{aligned}
$$

which proves both claims.

We remark that if $w, w^{\prime} \in \mathcal{W}^{j N}$ are distinct, then $\mathrm{K}_{w}$ may intersect $\mathrm{K}_{w^{\prime}}$. This is why we lift different sets $X_{w}$ to different heights.

For each $j=0,1, \ldots$ and each $w \in \mathcal{W}^{j N}$ define

$$
\mathcal{K}_{w}:=\mathrm{K}_{w} \times\left[\phi_{j}\left(x_{w}\right)-2 \operatorname{diam} X_{w}, \phi_{j}\left(x_{w}\right)+2 \operatorname{diam} X_{w}\right] \subset \mathbb{R}^{n+1} .
$$

We first claim that for all $j \in \mathbb{N}$, for all $w \in \mathcal{W}^{j N}$ and for all $u \in \mathcal{W}^{N}$,

$$
\mathcal{K}_{w u} \subset \mathcal{K}_{w} \quad \text { and } \quad \operatorname{dist}\left(\mathcal{K}_{w u}, \partial \mathcal{K}_{w}\right) \geq(8 \sqrt{n+1})^{-1} \operatorname{diam} \mathcal{K}_{w} .
$$

Let $z=\left(z_{1}, \ldots, z_{n+1}\right) \in \mathcal{K}_{w u}$. By (4.2) and the choice of $N$,

$$
\begin{aligned}
\left|z_{n+1}-\phi_{j}\left(x_{w}\right)\right| & \leq\left|z_{n+1}-\phi_{j+1}\left(x_{w u}\right)\right|+\left|\phi_{j}\left(x_{w}\right)-\phi_{j+1}\left(x_{w u}\right)\right| \\
& \leq 2 \operatorname{diam} X_{w u}+(4 k)^{-1}(k-1) \operatorname{diam} X_{w} \\
& \leq 2 \eta\left(2 \cdot 3^{-N}\right) \operatorname{diam} X_{w}+\frac{1}{4} \operatorname{diam} X_{w} \\
& \leq \frac{1}{2} \operatorname{diam} X_{w} .
\end{aligned}
$$

Therefore, $z \in \mathcal{K}_{w}$ and

$$
\operatorname{dist}\left(z, \partial \mathcal{K}_{w}\right) \geq \max \left\{\operatorname{dist}\left(\mathrm{K}_{w u}, \partial \mathrm{K}_{w}\right), 2 \operatorname{diam} X_{w}-\left|z_{n+1}-\phi_{j}\left(x_{w}\right)\right|\right\} \geq \frac{1}{2} \operatorname{diam} X_{w}
$$
and the claim follows.

Second, we claim that for all integers $j \geq 0$, all $w \in \mathcal{W}^{j N}$ and all distinct $u, u^{\prime} \in \mathcal{W}^{N}$,

$$
\operatorname{dist}\left(\mathcal{K}_{w u}, \mathcal{K}_{w u^{\prime}}\right) \geq\left(8 \eta\left(3^{N}\right) \sqrt{n+1}\right)^{-1} \operatorname{diam} \mathcal{K}_{w} .
$$

To prove (4.6), let $x \in \mathcal{K}_{w u}$ and $x^{\prime} \in \mathcal{K}_{w u^{\prime}}$. There are two cases to consider. 
Case 1. Suppose that $u, u^{\prime} \in \mathscr{E}_{i}^{w}$. Then $\phi_{j+1}\left(x_{w u}\right)=\phi_{j+1}\left(x_{w u^{\prime}}\right)$ and by the choice of $D$ we have

$$
\begin{aligned}
\left|x-x^{\prime}\right| & \geq\left|x_{w u}-x_{w u^{\prime}}\right|-\operatorname{diam} \mathcal{K}_{w u}-\operatorname{diam} \mathcal{K}_{w u^{\prime}} \\
& \geq \operatorname{dist}\left(X_{w u}, X_{w u^{\prime}}\right)-2 \max \left\{\operatorname{diam} \mathcal{K}_{w u}, \operatorname{diam} \mathcal{K}_{w u^{\prime}}\right\} \\
& \geq(D-8 \sqrt{n+1}) \max \left\{\operatorname{diam} X_{w u}, \operatorname{diam} X_{w u^{\prime}}\right\} \\
& =\max \left\{\operatorname{diam} X_{w u}, \operatorname{diam} X_{w u^{\prime}}\right\} .
\end{aligned}
$$

Case 2. Suppose that $u \in \mathscr{E}_{i}^{w}$ and $u^{\prime} \in \mathscr{E}_{i^{\prime}}^{w}$ with $i \neq i^{\prime}$. By the choice of $N$, we calculate the vertical difference of $x, x^{\prime}$

$$
\begin{aligned}
\left|x-x^{\prime}\right| & \geq\left|\phi_{j+1}\left(x_{w u}\right)-\phi_{j+1}\left(x_{w u^{\prime}}\right)\right|-2 \operatorname{diam} X_{w u}-2 \operatorname{diam} X_{w u} \\
& \geq(4 k)^{-1} \operatorname{diam} X_{w}-4 \max \left\{\operatorname{diam} X_{w u}, \operatorname{diam} X_{w u^{\prime}}\right\} \\
& \geq\left(\frac{1}{4 k \eta\left(2 \cdot 3^{-N}\right)}-4\right) \max \left\{\operatorname{diam} X_{w u}, \operatorname{diam} X_{w u^{\prime}}\right\} \\
& \geq \max \left\{\operatorname{diam} X_{w u}, \operatorname{diam} X_{w u^{\prime}}\right\} .
\end{aligned}
$$

In either case, (4.6) follows now from (4.2).

Third, by (4.2), we have that for all $j \in \mathbb{N}$, for all $w \in \mathcal{W}^{j}$ and for all $u \in \mathcal{W}^{N}$,

$$
\operatorname{diam} \mathcal{K}_{w u} \geq\left(2 \eta\left(3^{N}\right)\right)^{-1} \operatorname{diam} \mathcal{K}_{w} .
$$

Finally, by design of $\Phi$,

$$
\mathcal{K}_{w} \cap \Phi(X)=\Phi\left(X_{w}\right) \text { and } \operatorname{dist}\left(\Phi\left(X_{w}\right), \partial \mathcal{K}_{w}\right) \geq \operatorname{diam} X_{w} .
$$

For each $j=0,1, \ldots$ and $w \in \mathcal{W}^{j N}$, let $z_{w}$ be the center of $I_{w}$, define the cube

$$
\mathcal{Q}_{w}=\left[z_{w}-\frac{5}{6} 3^{-j N}, z_{w}+\frac{5}{6} 3^{-j N}\right] \times\left[-\frac{5}{6} 3^{-j N}, \frac{5}{6} 3^{-j N}\right]^{n},
$$

and let $g_{w}: \partial \mathcal{K}_{w} \rightarrow \partial \mathcal{Q}_{w}$ be an orientation preserving similarity map. By (4.5), (4.6) and (4.7), Proposition 3.3 applies and there exists $\Lambda>1$ depending only on $n, \eta$, (hence only on $n, c)$ such that $g_{w}$ extends as a $\left(\frac{\operatorname{diam} \mathcal{Q}_{w}}{\operatorname{diam} \mathcal{K}_{w}}, \Lambda\right)$-quasisimilarity homeomorphism of $\mathcal{K}_{w} \backslash\left(\bigcup_{u \in \mathcal{W}^{N}} \mathcal{K}_{w u}\right)$ onto $\mathcal{Q}_{w} \backslash\left(\bigcup_{u \in \mathcal{W}^{N}} \mathcal{Q}_{w u}\right)$.

Define now $G: \mathbb{R}^{n+1} \backslash \Phi(X) \rightarrow \mathbb{R}^{n+1} \backslash \mathcal{C}$ so that

(1) $G: \mathbb{R}^{n+1} \backslash \mathcal{K}_{\varepsilon} \rightarrow \mathbb{R}^{n+1} \backslash \mathcal{Q}_{\varepsilon}$ is a similarity,

(2) for each $w \in \mathcal{W}^{j N}$, the restriction of $G$ on $\mathcal{K}_{w} \backslash\left(\bigcup_{u \in \mathcal{W}^{N}} \mathcal{K}_{w u}\right)$ is $g_{w}$.

Since the diameters of cubes $\mathcal{K}_{w}$ and $\mathcal{Q}_{w}$ go to zero as $|w| \rightarrow \infty$, the map $G$ extends to a homeomorphism $G: \mathbb{R}^{n+1} \rightarrow \mathbb{R}^{n+1}$ mapping $\Phi(X)$ onto $\mathcal{C}$.

Let

$$
E=\mathcal{C} \cup \bigcup_{j \geq 0} \bigcup_{w \in \mathcal{W}^{j n}} \partial \mathcal{Q}_{w} .
$$

Then $E$ is a closed set of $\sigma$-finite $\mathcal{H}^{n}$-measure in $\mathbb{R}^{n+1}$ and for some $K \geq 1$ depending only on $c$ and $n, G^{-1}$ is $K$-quasiconformal on $\mathbb{R}^{n+1} \backslash E$. Therefore, by a theorem of Väisälä on removability of singularities [23, Theorem 35.1], $G^{-1}$ extends to a $K$-quasiconformal homeomorphism of $\mathbb{R}^{n+1}$, hence $G$ is quasiconformal. Set $F=G \circ \Phi$ and note that $F$ extends $f$. Therefore, $F(X)=\mathcal{C}$. 
4.1. Proof of Corollary $\mathbf{A}$, For the proof of Corollary $\mathrm{A}$, recall that a set $E \subset$ $[0,1]$ is porous if there exists $c \geq 1$ such that for any interval $I \subset[0,1]$, there exists an interval $J \subset I \backslash E$ of length $|J| \geq c^{-1}|I|$.

Proof of Corollary $\mathrm{A}$, Let $X \subset \mathbb{R}^{n}$ be bounded and $c$-uniformly disconnected. It now suffices to show that $X$ is contained in a compact uniformly perfect and uniformly disconnected set and Theorem 4.1 applies.

Replacing $X$ by $\bar{X}$, we may assume that $X$ is compact. By Theorem 1 in [15] (see also [15, p. 275] for discussion and [1, Theorem 3.8] for a more general statement), there exists an $\eta$-quasisymmetric map $f:[0,1] \rightarrow \mathbb{R}^{n}$ such that $X \subset f([0,1])$, $\{f(0), f(1)\} \subset X$, and $\eta$ depending only on $c$. Set $E=f^{-1}(X) \subset[0,1]$ which is uniformly disconnected and contains 0 and 1 .

For each component $I$ of $[0,1] \backslash E$, let $\phi_{I}: \mathbb{R} \rightarrow \mathbb{R}$ be a similarity that maps $[0,1]$ onto $\bar{I}$. Define now

$$
E^{\prime}:=E \cup \bigcup_{I} \phi_{I}(\mathcal{C})=\overline{\bigcup_{I} \phi_{I}(\mathcal{C})}
$$

where the union is over all components $I$ of $[0,1] \backslash X$. We claim that $E^{\prime}$ is uniformly perfect and uniformly disconnected. Assuming the latter, $X^{\prime}=f\left(E^{\prime}\right)$ is a compact uniformly perfect, and uniformly disconnected set that contains $X$. By Theorem $\mathrm{A}$, there exists an $\eta$-quasisymmetric map $F: \mathbb{R}^{n+1} \rightarrow \mathbb{R}^{n+1}$ with $F(X) \subset F\left(X^{\prime}\right)=\mathcal{C}$.

To prove the claim, recall that $\mathcal{C}$ is $C_{0}$-uniformly perfect and $C_{0}$-uniformly disconnected for some $C_{0}>1$.

To show that $E^{\prime}$ is uniformly perfect, fix $x \in E^{\prime}$ and $r \in(0,1)$. We claim that there exists universal $C \geq 1$ such that

$$
E^{\prime} \cap((x-r, x+r) \backslash(x-r / C, x+r / C)) \neq \emptyset .
$$

If $(x-r, x+r) \cap E=\emptyset$, then there exists $I$ as above such that $(x-r, x+r) \subset I$ and $(x-r, x+r) \cap E^{\prime}=(x-r, x+r) \cap \phi_{I}(\mathcal{C})$ and the claim is true for $C=C_{0}$. Suppose now that $(x-r, x+r) \cap E \neq \emptyset$. If there exists $z \in(x-r, x+r) \cap E$ with $|z-x| \geq r / 2$, then the claim is true for $C=2$. Suppose now that $(x-r / 2, x+r / 2) \cap E=$ $(x-r, x+r) \cap E \neq \emptyset$ and let $z \in[x, x+r / 2) \cap E$ such that $z-x$ is maximal. That is, $z$ is the left endpoint of a component $I$ as above and by the uniform perfectness of $\mathcal{C}$,

$$
\phi_{I}(\mathcal{C}) \cap\left(x+\left(2 C_{0}\right)^{-1} r, x+r\right) \supset \phi_{I}(\mathcal{C}) \cap\left(z+\left(2 C_{0}\right)^{-1} r, z+r / 2\right) \neq \emptyset
$$

and the claim holds true for $C=2 C_{0}$.

To show that $E^{\prime}$ is uniformly disconnected, by [15, Theorem 1], we need to show that $E^{\prime}$ is porous. Fix an interval $I \subset[0,1]$. The porosity of $E$ implies that there exists an interval $J^{\prime} \subset I \backslash E$ such that $\left|J^{\prime}\right| \geq|I| / c_{1}$ where $c_{1}$ depends only on $C$. Now, the porosity of $J^{\prime} \cap E^{\prime}$ (since it is a subset of a copy of $\mathcal{C}$ ) implies that there exists an interval $J \subset J^{\prime} \backslash E^{\prime}$ such that $|J| \geq\left|J^{\prime}\right| / c_{0}$ where $c_{0}$ depends only on $C_{0}$. Alltogether, $J \subset I \backslash E^{\prime}$ and $|J| \geq\left(c_{1} c_{0}\right)^{-1}|I|$.

\section{ACKNOWLEDGMENT}

We thank the anonymous referee whose comments and suggestions greatly improved the exposition of the paper. 


\section{REFERENCES}

[1] Matthew Badger and Vyron Vellis, Geometry of measures in real dimensions via Hölder parameterizations, J. Geom. Anal. 29 (2019), no. 2, 1153-1192, DOI 10.1007/s12220-0180034-2. MR.3935254

[2] Mario Bonk, Quasiconformal geometry of fractals, International Congress of Mathematicians. Vol. II, Eur. Math. Soc., Zürich, 2006, pp. 1349-1373. MR2275649

[3] Mario Bonk and Bruce Kleiner, Quasisymmetric parametrizations of two-dimensional metric spheres, Invent. Math. 150 (2002), no. 1, 127-183, DOI 10.1007/s00222-002-0233-z. MR.1930885

[4] Mario Bonk and Daniel Meyer, Quasiconformal and geodesic trees, Fund. Math. 250 (2020), no. 3, 253-299, DOI 10.4064/fm749-7-2019. MR4107537

[5] Robert J. Daverman, Decompositions of manifolds, AMS Chelsea Publishing, Providence, RI, 2007. Reprint of the 1986 original, DOI 10.1090/chel/362. MR2341468

[6] Guy David and Stephen Semmes, Fractured fractals and broken dreams, Oxford Lecture Series in Mathematics and its Applications, vol. 7, The Clarendon Press, Oxford University Press, New York, 1997. Self-similar geometry through metric and measure. MR 1616732

[7] Herbert Federer, Geometric measure theory, Die Grundlehren der mathematischen Wissenschaften, Band 153, Springer-Verlag New York Inc., New York, 1969. MR0257325

[8] Alastair N. Fletcher and Vyron Vellis, Decomposing multitwists, arXiv:2106.00054, 2021.

[9] Alastair N. Fletcher and Vyron Vellis, On uniformly disconnected Julia sets, Math. Z., posted online March 3, 2021. DOI 10.1007/s00209-021-02699-6, to appear in print.

[10] Alastair Fletcher and Jang-Mei Wu, Julia sets and wild Cantor sets, Geom. Dedicata 174 (2015), 169-176, DOI 10.1007/s10711-014-0010-3. MR3303046

[11] Juha Heinonen, Lectures on analysis on metric spaces, Universitext, Springer-Verlag, New York, 2001, DOI 10.1007/978-1-4613-0131-8. MR.1800917

[12] Tadeusz Iwaniec and Gaven Martin, Quasiregular semigroups, Ann. Acad. Sci. Fenn. Math. 21 (1996), no. 2, 241-254. MR 1404085

[13] Alexander S. Kechris, Classical descriptive set theory, Graduate Texts in Mathematics, vol. 156, Springer-Verlag, New York, 1995, DOI 10.1007/978-1-4612-4190-4. MR.1321597

[14] John M. Mackay and Jeremy T. Tyson, Conformal dimension, University Lecture Series, vol. 54, American Mathematical Society, Providence, RI, 2010. Theory and application, DOI 10.1090/ulect/054. MR2662522

[15] Paul MacManus, Catching sets with quasicircles, Rev. Mat. Iberoamericana 15 (1999), no. 2, 267-277, DOI 10.4171/RMI/256. MR.1715408

[16] Pekka Pankka and Jang-Mei Wu, Deformation and quasiregular extension of cubical Alexander maps, preprint, arXiv:1904.09095, 2019.

[17] Ch. Pommerenke, Uniformly perfect sets and the Poincaré metric, Arch. Math. (Basel) 32 (1979), no. 2, 192-199, DOI 10.1007/BF01238490. MR534933

[18] Dennis Sullivan, Hyperbolic geometry and homeomorphisms, Geometric topology (Proc. Georgia Topology Conf., Athens, Ga., 1977), Academic Press, New York-London, 1979, pp. 543555. MR537749

[19] Pekka Tukia, The planar Schönflies theorem for Lipschitz maps, Ann. Acad. Sci. Fenn. Ser. A I Math. 5 (1980), no. 1, 49-72, DOI 10.5186/aasfm.1980.0529. MR595177

[20] P. Tukia and J. Väisälä, Quasisymmetric embeddings of metric spaces, Ann. Acad. Sci. Fenn. Ser. A I Math. 5 (1980), no. 1, 97-114, DOI 10.5186/aasfm.1980.0531. MR595180

[21] P. Tukia and J. Väisälä, Lipschitz and quasiconformal approximation and extension, Ann. Acad. Sci. Fenn. Ser. A I Math. 6 (1981), no. 2, 303-342 (1982), DOI 10.5186/aasfm.1981.0626. MR658932

[22] Jeremy Tyson, Quasiconformality and quasisymmetry in metric measure spaces, Ann. Acad. Sci. Fenn. Math. 23 (1998), no. 2, 525-548. MR 1642158

[23] Jussi Väisälä, Lectures on n-dimensional quasiconformal mappings, Lecture Notes in Mathematics, vol. 229 Springer-Verlag, Berlin, 1971.

[24] Vyron Sarantis Vellis, Quasisymmetric spheres constructed over quasidisks, ProQuest LLC, Ann Arbor, MI, 2014. Thesis (Ph.D.)-University of Illinois at Urbana-Champaign. MR 3322047 
Department of Mathematics, The University of Tennessee, Knoxville, Tennessee 37916

Email address: vvellis@utk.edu 